\title{
Contextualizing the Content of Clinical Communications: A Learning Point
}

\author{
Ashish Walian $^{1}$ Rohan Magoon ${ }^{1}{ }^{10} \quad$ ItiShri ItiShri $^{1}$ \\ ${ }^{1}$ Department of Cardiac Anaesthesia, Atal Bihari Vajpayee Institute of \\ Medical Sciences (ABVIMS) and Dr. Ram Manohar Lohia Hospital, \\ New Delhi, India \\ ${ }^{2}$ Goa Medical College, Bambolim, Goa, India \\ J Card Crit Care 2021;5:263-264.
}

Suboptimal communication or inadequate exchange of important clinical information can be peculiarly perilous in subspecialties like cardiac anesthesia that are characterized by a highly predisposed working environment. ${ }^{1-3}$ While the fraternity acknowledges an effective communication to be at the cornerstone of minimizing the susceptibility to avoidable perioperative critical events, as George Bernard Shaw aptly puts it: the major problem with communication is precisely the illusion that it has taken place. ${ }^{1,4}$

The diversity in the experience of the communicating clinical workforce and the subjectivity of the communication mode compound the matter furthermore. Withstanding the above-mentioned fact, refinement of the objective communication skills of the young trainees continues to be an area of active interest. In this respect, the UK National Health Service (NHS) endorsed: Situation, Background, Assessment, Recommendation (SBAR) classifies as the most frequently cited tool for clinical communications. ${ }^{1}$ Devised by Doug Bonacum, the origins of the SBAR tool are linked to the US Navy with its implementation aimed at effectively communicating critical mission information regardless of the hierarchical factors. ${ }^{5}$

Despite inconsistencies in the literature on SBAR application, partly owing to issues like compliance and physiciandependence of the information transfer in heterogeneous settings, it is expected to enhance the completeness of the patient-related communication during face-to-face handovers among clinicians. ${ }^{1,5}$ This is heralded by the fact that the informative content needs to be closely backed by the contextual component wherein a SBAR approach can be of particular assistance. The former can be explained in the light
Raj Karhan Singh Kohli ${ }^{2}$

Address for correspondence ItiShri, MD, Department of Cardiac Anaesthesia, Atal Bihari Vajpayee Institute of Medical Sciences (ABVIMS) and Dr. Ram Manohar Lohia Hospital, Baba Kharak Singh Marg, New Delhi 110001, India (e-mail: iti.anesthesia@gmail.com).

of the following SBAR-based handoff of a patient manifesting a state of hemodynamic compromise:

- Situation: Difficulty in maintenance of hemodynamic stability while weaning from cardiopulmonary bypass (CPB) in a 2-year-old patient who has undergone a ventricular septal defect (VSD) closure.

- Background: The patient had a documented severe preoperative pulmonary artery hypertension (PAH, associated with a large VSD) and a prolonged aortic cross clamp and CPB time.

- Assessment: Hypotension with high right atrial pressures, desaturation amid an unexplained high airway pressure, and tachycardia with ischemic changes in right-sided leads suggest a setting of PAH crisis as the cause of hemodynamic instability. Assessment for the presence of PAH aggravating factors (acidosis, hypoxia, hypercarbia, and pain) accompanied by an echocardiographic assessment of PAH and concomitant right ventricular (RV) function.

- Recommendation: To break the vicious cycle of PAH(hypoxia, acidosis, hypercarbia)-PAH by augmenting the fractional inspired oxygenation, ensuring an adequate minute ventilation and correcting metabolic acidosis. Inotropic-pulmonary vasodilator infusions, like adrenaline and milrinone, to support the RV function and alleviate PAH. In refractory cases, call for team-help to initiate inhaled nitric oxide or extracorporeal membrane oxygenation.

While the SBAR model can prove to be instrumental in a structured communication between specialists, the need for a succinct nonspecialist based "go-between" communication published online

January 11, 2022
DOI https://doi.org/

10.1055/s-0041-1739529. ISSN 2457-0206.
(C) 2022. Official Publication of The Simulation Society (TSS), accredited by International Society of Cardiovascular Ultrasound (ISCU). All rights reserved.

This is an open access article published by Thieme under the terms of the Creative Commons Attribution-NonDerivative-NonCommercial-License, permitting copying and reproduction so long as the original work is given appropriate credit. Contents may not be used for commercial purposes, or adapted, remixed, transformed or built upon. (https://creativecommons.org/ licenses/by-nc-nd/4.0/)

Thieme Medical and Scientific Publishers Pvt. Ltd., A-12, 2nd Floor, Sector 2, Noida-201301 UP, India 
to seek requisite clinical assistance can also not be undermined. MacDougall-Davis et al propose a traffic-light colorcoded tool to flag the urgency of the clinical situation to minimize the possibility of the situational gravity from being lost-in-translation in a "go-between" communication. ${ }^{1}$

With the availability of lucid patient handover checklist tools like SBAR, there are ever-increasing viable options to provide an accurate, concise, consistent, and effective patient care transfer. Alongside the institutionalization of the protocols and practices, the standardization of the communication within the clinical framework is the need of the hour. The notion is strengthened by the literature on the role of organizational factors in influencing the patient safety. ${ }^{6}$ Moreover, this paradigm shift can be further perpetuated in the information technology age by supporting the development, incorporation, and operationalization of the computerized patient handover applications as a component of the electronic clinical record systems. ${ }^{7}$

Conflict of Interest

We do not have any conflict of interest, any commercial, or financial interest in this material and agree to abide by the rules of your journal regarding publication of this article.

\section{References}

1 MacDougall-Davis SR, Kettley L, Cook TM. The 'go-between' study: a simulation study comparing the 'Traffic Lights' and 'SBAR' tools as a means of communication between anaesthetic staff. Anaesthesia 2016;71(07):764-772

2 Magoon R. Precision cardiac anesthesia: welcome aboard!. J Cardiothorac Vasc Anesth 2020;34(09):2551-2552

3 Haynes AB, Weiser TG, Berry WR, et al; Safe Surgery Saves Lives Study Group. A surgical safety checklist to reduce morbidity and mortality in a global population. N Engl J Med 2009;360(05): 491-499

4 Cook TM, Woodall N, Frerk CFourth National Audit Project. Major complications of airway management in the UK: results of the Fourth National Audit Project of the Royal College of Anaesthetists and the Difficult Airway Society. Part 1: anaesthesia. Br J Anaesth 2011;106(05):617-631

5 Leonard M, Graham S, Bonacum D. The human factor: the critical importance of effective teamwork and communication in providing safe care. Qual Saf Health Care 2004;13(Suppl 1): i85-i90

6 Richter JP, McAlearney AS, Pennell ML. The influence of organizational factors on patient safety: examining successful handoffs in health care. Health Care Manage Rev 2016;41(01):32-41

7 Vawdrey DK, Stein DM, Fred MR, Bostwick SB, Stetson PD. Implementation of a computerized patient handoff application. AMIA Annu Symp Proc 2013;2013:1395-1400 